\title{
Non-inferiority of dose reduction versus standard dosing of TNF-inhibitors in axial spondyloarthritis
}

\author{
Jordi Gratacós ${ }^{1}$, Caridad Pontes ${ }^{2 *}$ D, Xavier Juanola ${ }^{3}$, Jesús Sanz ${ }^{4}$, Ferran Torres ${ }^{5}$, Cristina Avendaño ${ }^{6}$, \\ Antoni Vallano ${ }^{7}$, Gonzalo Calvo ${ }^{8}$, Eugenio de Miguel ${ }^{9}$, Raimon Sanmartí ${ }^{10}$ and The REDES-TNF investigators
}

\begin{abstract}
Objective: The objective was to determine if dose reduction is non-inferior to full-dose TNFi to maintain low disease activity (LDA) in patients already in remission with TNFi, in axial spondyloarthritis.

Methods: Randomized, parallel, non-inferiority, open-label multicentre clinical trial. Patients were eligible if they had axial spondyloarthritis and had been in clinical remission for $\geq 6$ months with any available TNFi (adalimumab, etanercept, infliximab, golimumab) at the dose recommended by product labelling. Patients were randomized by automated central allocation to continue the same TNFi dose schedule, or to reduce the dose by roughly half according to the protocol. The main outcome was the proportion of subjects with LDA after 1 year. Serious adverse reactions or infections were recorded.

Results: The trial stopped due to end of the funding period, after 126 patients were randomized; 113 patients (84.1\% male, mean age (SD) 45.6 (13.0) years) were included in the main per-protocol subset. Non-inferiority was concluded for LDA at 1 year (47/55 (83.8\%) patients in the full-dose and 48/58 (81.3\%) patients in the reduced-dose arm, adjusted difference (95\% Cl) $-2.5 \%$ ( $-16.6 \%$ to $11.7 \%)$ ). Serious adverse reactions or infections were reported in $7 / 62$ patients (11.3\%) assigned to full dose and 2/61 patients (3.3\%) assigned to reduced dose ( $p$ value $=0.164$ ).
\end{abstract}

Conclusion: In patients with ankylosing spondylitis in clinical remission for at least 6 months, dose reduction is non-inferior to full TNF inhibitor doses to maintain LDA after 1 year. Serious adverse events may be less frequent with reduced doses.

Trial registration: EU Clinical Trials Registry, EudraCT 2011-005871-18 and ClinicalTrials.gov, NCT01604629.

Keywords: Spondyloarthritis, Dose-tapering, Non-inferiority, TNF inhibitors

\section{Introduction}

Spondyloarthritis (SpA), a group of rheumatic diseases that share immunogenic, clinical and radiological characteristics, are classified as axial (axSpA) or peripheral by the Assessment of Spondyloarthritis International Society (ASAS). Ankylosing spondylitis (AS) is the main disease within SpA, with a prevalence of $0.4-0.9 \%$ in Caucasians, and marked differences by race, prevalence of HLA B27, and geographical area $[1,2]$. Despite treatment, many Patients with SpA have impaired quality of life and work disability [3, 4]. SpA management aims to

\footnotetext{
* Correspondence: caridad.pontes@uab.cat

${ }^{2}$ Clinical Pharmacology Unit, Hospital de Sabadell, Institut Universitari Parc

Taulí, Universitat Autònoma de Barcelona, c/Taulí n¹, 08208 Sabadell,

Barcelona, Spain

Full list of author information is available at the end of the article
}

achieve and maintain clinical remission or low disease activity (LDA) [5]. The first step is non-steroidal anti-inflammatory drugs (NSAIDs) and physical therapy, although $60 \%$ of patients obtain no clinical benefit [6]. Disease-modifying anti-rheumatic drugs (DMARDs) are not deemed efficacious, but tumour necrosis factor inhibitors (TNFi) can induce and sustain long-term clinical remission in patients not responding to NSAIDs [7]. However, the optimal duration and dose for long-term treatment remain unclear, and the recommended TNFi doses are the same for treatment of acute and chronic disease. Although the risk-benefit is favourable, prolonged treatment with TNFi chronically suppresses the immune response, increasing the risk of serious infection dose-dependently, especially in frail patients. It is unclear whether chronic use may increase malignancies 
and neurological demyelinating diseases [8-10]. Finally, TNFi are expensive and a cost burden.

Discontinuation of TNFi after achieving clinical remission is followed by early relapse in most cases [11-13]. However, lower doses of TNFi may be sufficient in patients with LDA and low levels of inflammatory mediators, according to uncontrolled or observational studies $[14,15]$. Some clinical guidelines recommend empirical dose reductions despite a lack of robust supportive evidence [16]. Likewise, although drug-level monitoring may help physicians optimize and prevent overtreatment in patients with rheumatoid arthritis treated with TNFi [17], there are no conclusive data on useful biomarkers for the monitoring of TNFi in AS [18].

The objective of this study was to determine the suitability of dose reduction for long-term treatment with TNFi in axSpA and potential predictors of poor clinical response. The main study hypothesis was that in patients with axSpA in persistent clinical remission with TNFi, reduced TNFi doses would be not inferior to full doses, as assessed by the proportion of patients not reaching LDA criteria for changing treatments. In addition, we analysed pre and post withdrawal drug levels, and markers of inflammation and bone markers in a subset.

\section{Patients and methods}

A prospective multicentre, parallel, controlled and randomized open-label clinical trial was conducted at 22 Spanish centres. Patients were eligible if they met criteria for axSpA according to the ASAS classification criteria [19], on treatment with the recommended doses of commercially available TNFi (infliximab, adalimumab, etanercept or golimumab) at the time of the study, and in sustained clinical remission defined as Bath Ankylosing Spondylitis Disease Activity Index $(\mathrm{BASDAI}) \leq 2$, no clinically active arthritis or enthesitis and C-reactive protein (CRP) equal to or higher than the upper limit of normality for $\geq 6$ months. Exclusion criteria were secondary SpA or predominantly peripheral arthritis, comorbidity interfering with the clinical assessment, pregnancy and breast feeding. Before any study-related procedure, patients were given comprehensive information on the study objectives, procedures and risks, and written informed consent was obtained from all patients.

\section{Randomization and treatments}

Eligible patients were randomized to receive either the full recommended TNFi dose or a reduced dose according to an agreed protocol supported by clinical practice (Table 1). Patients were screened, and information on previous TNFi treatment, clinical activity and other eligibility criteria entered in the electronic case report form (eCRF). Patients were automatically randomized by central allocation according to a list generated using SAS PROC PLAN v9.2 (SAS Institute Inc., Cary, NC, USA) with a 1:1 assignment ratio between arms, stratifying by prior TNFi treatment in blocks of four elements. The randomization list was loaded to a separate module of the eCRF so that the module automatically assigned the lowest sequential number available within the randomization stratum. An auditable registry of the date, time and other variables related to stratification and treatment assignment was kept. Patients and the study investigators were not blinded to treatment once assigned.

\section{Clinical outcomes}

The main clinical endpoint was the percentage of patients with LDA (BASDAI score $<4$, plus physician global assessment $<4$, patient global assessment $<4$ and nocturnal axial pain $<4$ as assessed on a $0-10$ visual analogue scale (VAS)) at one year. The key secondary endpoint was the proportion of patients who remained in clinical remission (BASDAI $<=2$, physician global assessment $<=2$, and patient global assessment $<=2$ with serum CRP below the upper limit of normality) at 1 year. Other secondary objectives were the usual axSpA clinical outcomes (Ankylosing Spondylitis Disease Activity Score (ASDAS)-CRP, ASAS response criteria, BASDAI, functional assessment as measured by Bath Anklylosing Spondylitis Functional Index (BASFI) and quality of life as measured by Ankylosing Spondylitis Quality of Life (ASQoL)). Relapses defined according to ASAS group, based on pain, BASDAI and ASDAS-CRP [20] were calculated a posteriori on recorded data. Adverse events, serious adverse reactions requiring hospitalization and/ or treatment withdrawal, and serious infections requiring systemic antibiotic treatment and/or hospitalization were recorded.

Table 1 Study treatments

\begin{tabular}{lllll}
\hline Anti-TNF drug & Posology according to SPC & Route & Full-dose group & Reduced-dose group \\
\hline Adalimumab & $40 \mathrm{mg}$ every 2 weeks & SC & $40 \mathrm{mg}$ every 2 weeks & $40 \mathrm{mg}$ every 3 weeks \\
Etanercept & $25-50 \mathrm{mg}$ every 3-7 days & SC & $25 \mathrm{mg}$ every 3 days or & $50 \mathrm{mg}$ every 10 days \\
Golimumab & $50 \mathrm{mg}$ every month & SC & $50 \mathrm{mg}$ every 7 days & $50 \mathrm{mg}$ every 6 weeks \\
Infliximab & $5 \mathrm{mg} /$ Kg every 6-8 weeks & IV & $5 \mathrm{mg} / \mathrm{Kg}$ every 6-8 weeks & $3 \mathrm{mg} / \mathrm{Kg}$ every 8 weeks \\
\hline
\end{tabular}

SPC Summary of Product Characteristics, SC subcutaneous, IV intravenous 
Blood samples for the measurement of TNFi, anti-drug antibodies and inflammatory mediators were collected within the $24 \mathrm{~h}$ before TNFi injection in 18 participating centres. The number of samples requested were $\geq 2$ per patient, separated by $\geq 90$ days when feasible. Samples were frozen and stored at $-80^{\circ} \mathrm{C}$ until shipment at the end of the study.

Inflammatory mediators were assayed at Laboratori de Recerca - I3PT (Sabadell, Spain). Plasma levels of TNF alpha, IL-6, sclerostin (SOST) and Dickkopf-related protein 1 (DKK-1) were determined using a Luminex multiplex immunoassay (Merck-Millipore, Darmstadt) (accuracy $=82$ $97 \%$, inter-assay and intra-assay precision $=<15 \%$ and $<$ 10\%, respectively); high-sensitivity CRP (hs-CRP) using a Luminex immunoassay (Merck-Millipore, Darmstadt) (accuracy $=93 \%$, inter-assay and intra-assay precision $=<15 \%$ and $<10 \%$, respectively); plasma calprotectin levels using Calprolab ELISA test HRP (Calpro, Lysaker) (inter-assay and intra-assay precision $<7 \%$ and $<6 \%$, respectively).

Drug and anti-drug antibody (ADA) levels were determined by assay at the Immunology Department - Hospital La Paz (Madrid, Spain). TNFi was measured by commercially available capture ELISA kits, (Promonitor, Progenika, Derio, Vizcaya), with cutoff values established using serum from 150 healthy blood donors and 100 patients with RA who were naive to biological drugs. The positivity threshold was mean optical density +6 standard deviations: positive levels were infliximab $>0.035 \mu \mathrm{g} /$ $\mathrm{ml}$, adalimumab $>0.024 \mu \mathrm{g} / \mathrm{ml}$, golimumab $>0.022 \mu \mathrm{g} /$ $\mathrm{ml}$ and etanercept $>0.035 \mu \mathrm{g} / \mathrm{ml}$. ADAs were assayed only in samples with no detectable TNFi, using a home-made, two-site (bridging) ELISA.

\section{Visit schedule}

Visits were scheduled according to routine clinical practice, although recommended intervals between visits were $8-16$ weeks [21]; a mandatory visit was required 1 year after randomization for the main assessment. Follow up continued until the end of study, change in biological treatment, withdrawal of informed consent or loss to follow up. NSAID treatment and dose modification was permitted throughout the study and was recorded. The study protocol has been reported in full elsewhere [22].

\section{Sample size and statistical methods}

The estimated proportion of patients at LDA after 1 year of treatment with full-dose TNFi was $87 \%$ in patients who were in stable remission for 4 months [23]. A non-inferiority margin of $17 \%$ was established, and protection against type I error $=2.5 \%$ (unilateral) and against type II error $=20 \%$, being the estimated sample size to test the non-inferiority hypothesis of the reduced-dose strategy with respect to the full-dose strategy of 85 patients per arm $[24,25]$. The non-inferiority (delta margin) of $17 \%$ was prospectively set based on the consensus on clinical relevance reached by the rheumatologists involved, who decided that a proportion of patients with acceptable control $<70 \%$ after 1 year would severely discourage the use of dose reduction [22]. To ensure the reliability of the study, the lower confidence interval (CI) in the full-dose arm had to be > $60 \%$ to confirm non-inferiority, in order to ensure that the control treatment had been reasonably effective [22].

A per-protocol set (PPS) for analysis was the pre-defined primary population for this non-inferiority study, following a conservative approach where any potential differences can be maximized. The principal endpoint and the key secondary endpoints were also tested in the full analysis set (FAS) population for sensitivity. The principal and key secondary end-points were assessed by estimating the between-treatment risk differences after 1 year of randomization and checking these against the pre-defined non-inferiority margin (delta $(\delta))$ of $17 \%$. Rates and risk differences were estimated using log-binomial regression including treatment and the factors used to stratify assignment. The effects of baseline clinical and inflammatory parameters on the results were evaluated, and the heterogeneity of the main results checked for stratification factors. A detailed statistical analysis plan was issued and approved on $25^{\text {th }}$ September 2014. The statistical analysis was run in the SAS System (SAS Institute Inc., Cary, NC, USA) v9.2. The detailed statistical methods have been reported elsewhere [22].

\section{Results}

From $6^{\text {th }}$ July 2012 to $15^{\text {th }}$ May 2014, 157 patients were screened. Due to recruitment delays and time restrictions on funding, recruitment was closed after 126 patients had been randomized; the last patient visit was on $10^{\text {th }}$ June 2015. Figure 1 shows the number of patients assigned to each treatment arm (FAS), lost to follow up during the study (total of three patients) and the reasons for exclusion from the PPS main analysis set. Baseline participant characteristics were similar between groups (Table 2). Although the protocol allowed inclusion of patients with axSpA, all included patients fulfilled criteria for AS.

The main study result fulfilled the objective of non-inferiority, with a proportion $(95 \% \mathrm{CI})$ of patients with LDA at one year of randomization of $83.8 \%$ (64.8$102.7 \%)$ in the full-dose arm and $81.3 \%(62.8-99.8 \%)$ in the reduced-dose arm, and absolute difference in LDA at 1 year between treatment groups of $-2.5 \%$ (95\% CI of 16.6 to $11.7 \%$ ) in the pre-defined PPS population (Fig. 2a and c). The lower bound of CI for the estimate in the control arm was $>60 \%$, fulfilling the pre-specified 


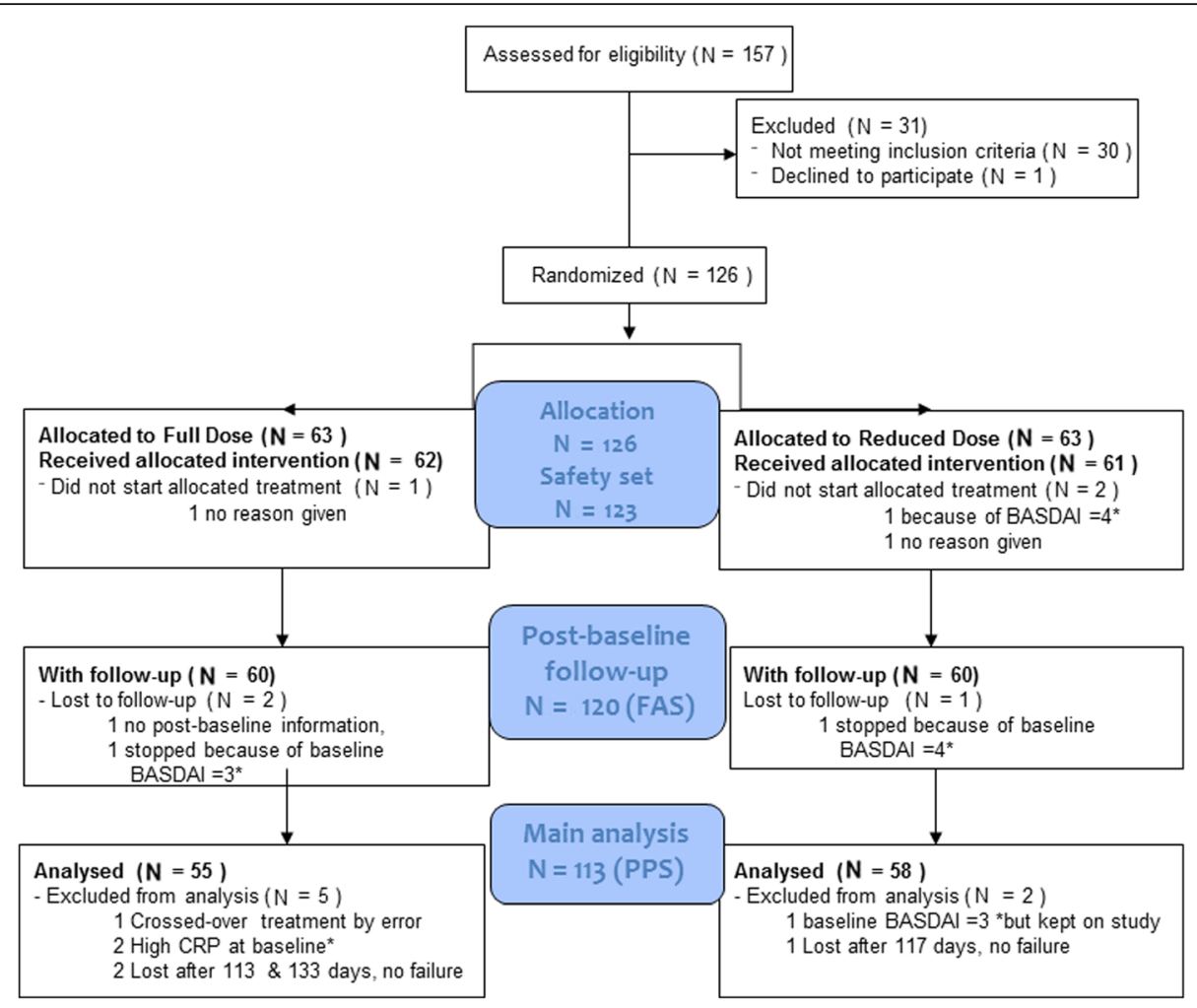

Fig. 1 Disposition of patients. FAS, full analysis; PPS, per-protocol main analysis set; BASDAI, Bath Ankylosing Spondylitis Disease Activity Index; CRP, C-reactive protein. ${ }^{*}$ Not meeting inclusion criteria for remission (BASDAI $\leq 2$ and CRP $\leq$ upper limit of normality)

validation criteria. The percentage $(95 \% \mathrm{CI})$ of patients in clinical remission (the key secondary endpoint) at one year of randomization was $83.7 \%(64.7-102.7 \%)$ in the full-dose arm and $78.2 \%(59.7-96.8 \%)$ in the reduced-dose arm, with absolute differences between treatment groups of $-5.5 \%(-20.6$ to $9.7 \%)(p=0.480)$ (Fig. 2a and c). The sensitivity analysis in the FAS population showed similar estimates for the differences between the two arms for the primary and key secondary end-points (Fig. 2b and d). No significant differences were observed between treatment arms for any of the secondary variables (Table 3 ).

At least one blood sample was available from each of 55 subjects (29 in the full-dose group and 26 in the reduced-dose group) for assessment of plasma levels of inflammatory biomarkers and drugs (Additional file 1: Table S1). All samples tested for TNFi levels were positive, and thus no ADA levels were determined.

Amongst clinical variables at baseline, BASFI, ASQoL and the type of TNFi were all significantly associated with the probability of LDA after 1 year in univariate models, but only the type of TNFi and BASFI remained significant in multivariate models. The treatment effect (dose group assigned) showed no significant heterogeneity according to the BASFI ( $p=0.5244)$ or type of TNFi $(p=0.887)$, thus suggesting that the predictive value of BASFI and type of
TNFi on the outcome is independent of the assigned dose schedule (full dose or reduced dose) (Additional file 1: Table S2). Considering inflammatory biomarkers and drug plasma levels, only hs-CRP levels were higher in subjects without LDA at 1 year (Additional file 1: Table S3). Univariate models including both clinical and analytical biomarkers had poor predictive value, with the lower CI for the ROC AUC always < 0.62 ; multivariate methods were unfeasible as no combination of factors improved the univariate models (Additional file 1: Table S3).

Of the 126 randomized patients, serious and related adverse events or infections were reported in $7 / 62$ patients $(11.3 \%)$ in the full-dose group and $2 / 61(3.3 \%)$ patients in the reduced-dose group $(p$ value $=0.164)$ (Table 3). A total of 34 infections were reported by 26 subjects, among whom 15 infections were reported by $11 / 62$ subjects $(17.7 \%)$ in the full-dose group and 4 infections were reported by $3 / 61$ subjects $(4.9 \%)$ in the reduced-dose group as being at least possibly related to treatment (Additional file 1: Table S4). Treatment was discontinued in 6/62 (9.7\%) and 13/61 patients (21.3\%) in the full-dose and reduced-dose groups, respectively ( $p$ value $=0.086)$, among whom $3 / 62(4.8 \%)$ in the full-dose and $8 / 61(13.1 \%)$ in the reduced-dose group had treatment discontinued due to poor disease control, according to physician judgment ( $p$ value $=0.204$ ) . 
Table 2 Baseline characteristics of patients

\begin{tabular}{|c|c|c|c|c|c|c|}
\hline \multirow[t]{2}{*}{ Baseline characteristics } & \multicolumn{3}{|l|}{ Full analysis set } & \multicolumn{3}{|l|}{ Per-protocol set } \\
\hline & $\begin{array}{l}\text { Full dose } \\
(n=60)\end{array}$ & $\begin{array}{l}\text { Dose reduction } \\
(n=60)\end{array}$ & $\begin{array}{l}\text { Total } \\
(n=120)\end{array}$ & $\begin{array}{l}\text { Full dose } \\
(n=55)\end{array}$ & $\begin{array}{l}\text { Dose reduction } \\
(n=58)\end{array}$ & $\begin{array}{l}\text { Total } \\
(n=113)\end{array}$ \\
\hline Gender (male), n (\%) & $53(88.3)$ & $49(81.7)$ & $102(85.0)$ & $48(87.3)$ & $47(81.0)$ & $95(84.1)$ \\
\hline Age (years), mean (SD) & $46.2(13.7)$ & $43.7(12.4)$ & $44.9(13.1)$ & $47.2(13.6)$ & $43.6(12.4)$ & $45.6(13.0)$ \\
\hline BMI, mean (SD) & $25.9(3.4)$ & $25.8(3.8)$ & $25.9(3.6)$ & $25.8(3.4)$ & $25.9(3.8)$ & $25.9(3.6)$ \\
\hline Years from diagnosis, median $\left(\mathrm{P}_{25}, \mathrm{P}_{75}\right)$ & $10.4(7.1,20.8)$ & $9.3(5.2,17.6)$ & $10.0(5.9,19.8)$ & $10.4(7.1,22.6)$ & $9.3(5.0,19.0)$ & $10.0(5.9,20.3)$ \\
\hline ASAS criteria for sacroilitis, $n(\%)$ & $58(96.7)$ & $59(98.3)$ & $117(97.5)$ & $53(96.4)$ & $57(98.3)$ & $110(97.3)$ \\
\hline ASDAS-CRP, median $\left(\mathrm{P}_{25}, \mathrm{P}_{75}\right)$ & $0.7(0.5,1.1)$ & $0.7(0.5,1.1)$ & $0.7(0.5,1.1)$ & $1.1(0.8,3.5)$ & $1.0(0.7,1.9)$ & $1.1(0.7,2.0)$ \\
\hline BASDAl, median $\left(P_{25}, P_{75}\right)$ & $1.0(0.6,1.7)$ & $1.0(0.2,1.4)$ & $1.0(0.4,1.6)$ & $1.0(0.6,1.7)$ & $1.0(0.2,1.4)$ & $1.0(0.4,1.6)$ \\
\hline VAS nocturnal axial pain, mean (SD) & $0.85(1.0)$ & $1.03(1.16)$ & $0.94(1.09)$ & $0.84(1.01)$ & $1.02(1.15)$ & $0.93(1.08)$ \\
\hline IGA, median $\left(P_{25}, P_{75}\right)$ & $1.0(0.0,2.0)$ & $1.0(0.0,1.0)$ & $1.0(0.0,1.0)$ & $1.0(0.0,2.0)$ & $1.0(0.0,1.0)$ & $1.0(0.0,1.0)$ \\
\hline PGA, median $\left(P_{25}, P_{75}\right)$ & $1.0(0.0,2.0)$ & $1.0(0.0,2.0)$ & $1.0(0.0,2.0)$ & $1.0(0.0,2.0)$ & $1.0(0.0,1.0)$ & $1.0(0.0,1.0)$ \\
\hline \multicolumn{7}{|l|}{ Current TNFi, n (\%) } \\
\hline Adalimumab & $24(40.0)$ & $23(38.3)$ & $47(39.2)$ & $22(40.0)$ & $22(37.9)$ & $44(38.9)$ \\
\hline Etanercept & $21(35.0)$ & $20(33.3)$ & $41(34.2)$ & $19(34.5)$ & $19(32.8)$ & 38 (33.6) \\
\hline Golimumab & $4(6.7)$ & $5(8.3)$ & $9(7.5)$ & $4(7.3)$ & $5(8.6)$ & $9(8.0)$ \\
\hline Infliximab & $11(18.3)$ & $12(20.0)$ & $23(19.2)$ & $10(18.2)$ & $12(20.7)$ & $22(19.5)$ \\
\hline \multicolumn{7}{|l|}{$\mathrm{N}$ of previous TNFi, $n(\%)$} \\
\hline None & $50(83.3)$ & $44(73.3)$ & $94(78.3)$ & $46(83.6)$ & $42(72.41)$ & $88(77.9)$ \\
\hline One & $10(16.7)$ & $11(18.3)$ & $21(17.5)$ & $9(16.4)$ & $11(19.0)$ & $20(17.7)$ \\
\hline Two & $0(0.0)$ & $5(8.3)$ & $5(4.2)$ & $0(0.0)$ & $5(8.6)$ & $5(4.4)$ \\
\hline NSAID use, $n(\%)$ & $16(26.7)$ & $14(23.3)$ & $28(24.8)$ & $15(27.3)$ & $13(22.4)$ & $28(24.8)$ \\
\hline
\end{tabular}

$n$ number, SD standard deviation, $P 25$ percentile 25, P75 percentile 75, BMI body mass index, ASAS Assessment of Spondyloarthritis International Society, ASDASCRP Ankylosing Spondylitis Disease Activity Score including C-reactive protein, BASDAl Bath Ankylosing Spondylitis Disease Activity Index; VAS patient's rating of nocturnal axial pain by visual analogue scale ranging from 0 (none) to 10 (worst), PGA Patient Global Assessment of disease activity rated from 0 (best) to 10 (worst), IGA Investigator's Global Assessment of disease activity rated from 0 (best) to 10 (worst), TNFi TNF inhibitor, NSAID non-steroidal anti-inflammatory drug

\section{Discussion}

This trial has shown that a dose-reduction strategy may be non-inferior to full-dose treatment in patients with AS in persistent clinical remission with TNFi, in terms of maintenance of LDA after 1 year. In the reduced-dose group, less serious and related adverse events or infections were observed, but also more withdrawals due to insufficient disease control, although not statistically significant.

Observational studies have reported that complete TNFi discontinuation in patients with AS is not feasible [16] despite $60-80 \%$ of patients have LDA at $12-21$ months after TNFi dose reduction [19, 26, 27]. However, these studies were uncontrolled or retrospective and sometimes had a small sample size, wide inclusion criteria and many dose schedules, leading to substantial heterogeneity that limited the generalizability of any dose-reduction recommendation.

Our results show that more than $80 \%$ and $77 \%$ of patients with AS treated with TNFi had LDA or remission at 1 year after randomization to standard or reduceddose schedules, respectively, in a pragmatic setting, with high external validity. Clear correlation has been reported in patients with AS between BASDAI, BASFI or ASDAS-CRP and disease activity, function and cost-effectiveness of treatments [28-30]. Our study has shown similar results in both randomized arms for these variables. No differences were observed between the groups in the frequency of flares, and no substantial changes in the proportion of NSAID treatments were observed during follow up in either TNFi regimen. However, despite the overall small number of early study withdrawals due to poor disease control, these were numerically higher in the reduced-dose group. This observation has to be interpreted in the context of an open-label study, and considering which the clinical options were in the case of observing worse clinical scores: in the reduced-dose arm, the options included returning to full-dose schedule, while in the full-dose group the options were more aggressive, either dose intensification or switching.

Since there are no universally accepted criteria for remission in AS, we used strict criteria at baseline, and randomized only patients with persistent and maintained clinical remission during the last 6 months while on TNFi [31]. In the absence of an alternative internationally agreed 


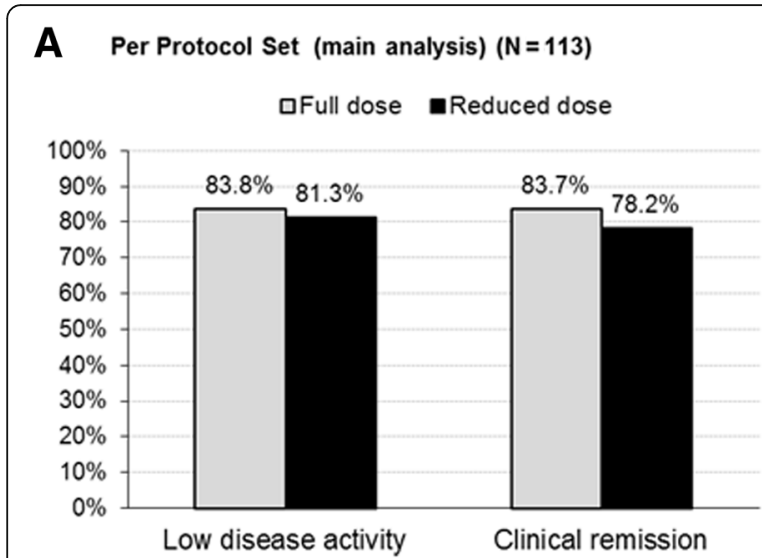

C Per Protocol Set (main analysis) $(\mathrm{N}=113)$

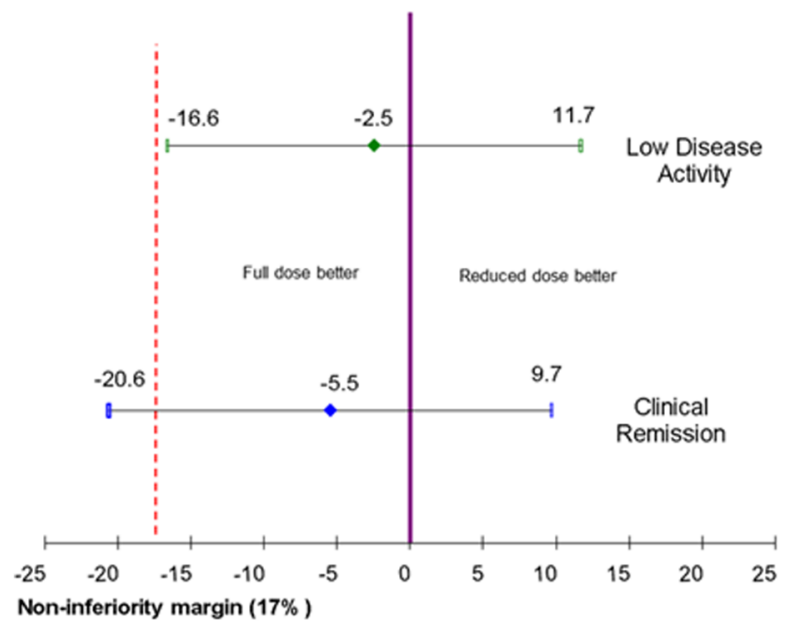

B Full Analysis Set $(\mathrm{N}=120)$

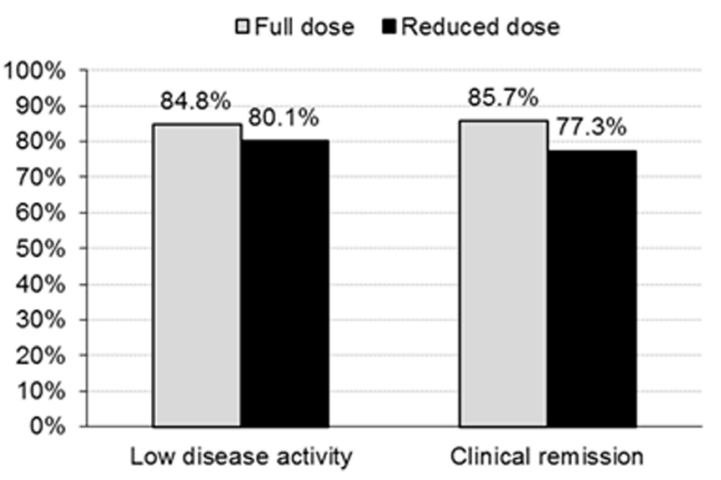

D Full Analysis Set $(N=120)$

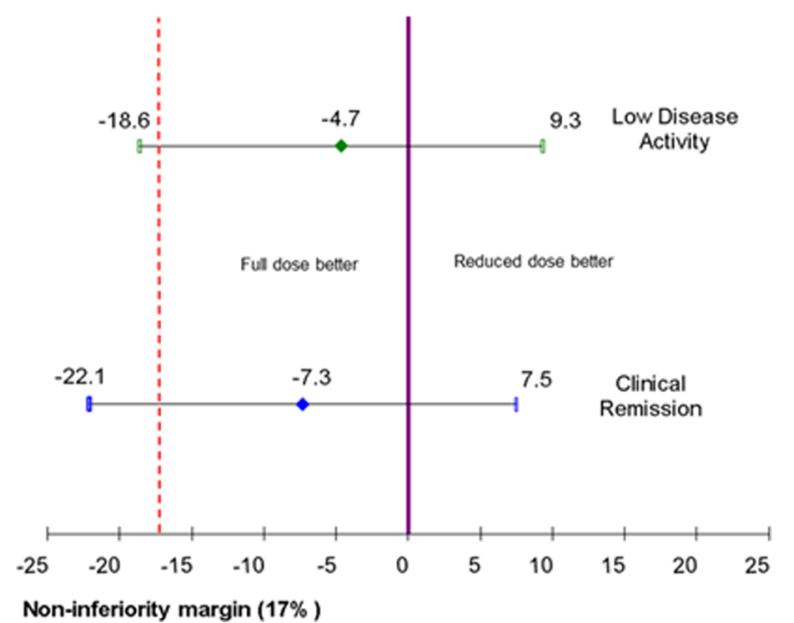

Fig. 2 Proportion of patients with low disease activity and clinical remission at 12 months. a Proportion of subjects with low disease activity and clinical remission at 12 months, per-protocol subset (main analysis). b Proportion of subjects with low disease activity and clinical remission at 12 months, intention-to-treat subset. c Adjusted differences between groups and non-inferiority testing for low disease activity and clinical remission, per-protocol subset (main analysis). $\mathbf{d}$ Adjusted differences between groups and non-inferiority testing for low disease activity and clinical remission, intention-to-treat subset. Low disease activity was defined by Bath Ankylosing Spondylitis Disease Activity Index (BASDAl) $<4$ and Physician Global Assessment $<4$, Patient Global Assessment $<4$ and axial pain at night $<4$. Clinical remission was defined by BASDAl $\leq 2$, Physician Global Assessment $\leq 2$ and Patient Global Assessment $\leq 2$

consensus on thresholds for response and control at the time of the study design, we used the Spanish Society of Rheumatology definition for low disease activity [16]. The definition was reflective of a consensus on the clinical therapeutic goal of treatment requiring action if not met. The definition was well-known, widely accepted and applied in clinical practice by rheumatologists in Spain.

The standardized dose-reduction protocol included lengthening the dosing interval by $50 \%$ for all subcutaneous TNFi and reducing the infliximab dose from 5 to 3 $\mathrm{mg} / 8$ week, according to reported data [15]. This allowed a reduction in the heterogeneity of drug regimes, a limitation reported by other studies [27], although it did not allow evaluation of the potential benefit of additional patient-tailored dose reductions.
Most patients were on etanercept and adalimumab, with $<20 \%$ of patients on infliximab, consistent with previous reports [12]. Randomization was stratified by drug to ensure comparability of groups; subgroup analysis based on stratification factors suggested that infliximab was associated with a higher risk of failure, although no interaction with the randomized strategy was observed, and thus the risk was similar regardless of whether doses were reduced or not. Considering that patients were not randomized to a given $\mathrm{TNFi}$, but to the dosing strategy, this observation is of limited value for causality assessment, and indication bias cannot be discarded. Similarly, although observational data suggest that dose reduction may be easier with etanercept [14], we found no differences for this stratum. 
Table 3 Secondary endpoints

\begin{tabular}{|c|c|c|c|c|}
\hline \multicolumn{5}{|c|}{ Efficacy secondary variables at 1 year } \\
\hline Variables & Full dose $(n=55)$ & Dose reduction $(n=58)$ & Differences between groups & $P$ value \\
\hline ASDAS-CRP $<1.3^{\mathrm{a}}$ & $61.4 \%[47.3 \% ; 75.5 \%]$ & $53.5 \%$ [39.7\%; 67.3\%] & $7.8 \%[-10.0 \% ; 25.8 \%]$ & 0.389 \\
\hline ASDAS-CRP relapse $e^{a}$ & $6.6 \%[-5.4 \% ; 18.7 \%]$ & $12.7 \%[1.4 \% ; 24.0 \%]$ & $-6.1 \%[-25.2 \% ; 12.9 \%]$ & 0.529 \\
\hline Relapse BASDAI-VAS ${ }^{a}$ & $15.9 \%[-3.1 \% ; 34.9 \%]$ & $10.4 \%[-9.1 \% ; 29.8 \%]$ & $5.5 \%[-12.3 \% ; 23.4 \%]$ & 0.545 \\
\hline Relapse SER ${ }^{\mathrm{a}}$ & $6.4 \%[-13.0 \% ; 25.8 \%]$ & $10.1 \%[-7.9 \% ; 28.1 \%]$ & $-3.7 \%[-19.2 \% ; 11.8 \%]$ & 0.638 \\
\hline NSAIDs use ${ }^{a}$ & $18.3 \%[-1.2 \% ; 37.7 \%]$ & $20.6 \%[1.5 \% ; 39.8 \%]$ & $-2.4 \%[-18.9 \% ; 14.2 \%]$ & 0.779 \\
\hline ASDAS-CRP score ${ }^{b}$ & $1.1(0.1)[0.9 ; 1.3]$ & $1.1(0.1)[0.9 ; 1.3]$ & $0.0(0.2)[-0.3 ; 0.3]$ & 0.783 \\
\hline BASDAl $^{\mathrm{b}}$ & $1.4(0.2)[1.1 ; 1.7]$ & $1.4(0.2)[1.1 ; 1.7]$ & $-0.0(0.2)[-0.5 ; 0.4]$ & 0.890 \\
\hline VAS night axial pain ${ }^{\mathrm{b}}$ & $1.4(0.2)[1.0 ; 1.8]$ & $1.1(0.2)[0.7 ; 1.6]$ & $0.3(0.3)[-0.3 ; 0.9]$ & 0.337 \\
\hline$P G A^{b}$ & $1.6(0.2)[1.2 ; 2.0]$ & $1.6(0.2)[1.1 ; 2.0]$ & $0.0(0.3)[-0.6 ; 0.6]$ & 0.962 \\
\hline$I G A^{b}$ & $1.1(0.2)[0.8 ; 1.4]$ & $1.1(0.2)[0.8 ; 1.4]$ & $-0.0(0.2)[-0.4 ; 0.40]$ & 0.923 \\
\hline $\mathrm{BASFI}^{\mathrm{b}}$ & $1.7(0.2)[1.2 ; 2.1]$ & $1.8(0.2)[1.3 ; 2.3]$ & $-0.2(0.3)[-0.8 ; 0.5]$ & 0.616 \\
\hline $\mathrm{ASQOL}^{\mathrm{b}}$ & $2.3(0.5)[1.2 ; 3.3]$ & $2.2(0.6)[1.0 ; 3.3]$ & $0.1(0.7)[-1.3 ; 1.5]$ & 0.858 \\
\hline \multicolumn{5}{|c|}{ Safety secondary variables } \\
\hline Adverse event or infections & Full dose $(n=62)$ & Dose reduction $(n=61)$ & Differences between groups & $P$ value \\
\hline Any $^{c}$ & 22 (35.5\%) [23.6\%;47.4\%] & 17 (27.9\%) [16.6\%;39.1\%] & $(7.6 \%)[-8.8 \% ; 24.0 \%]$ & 0.439 \\
\hline Related $^{c}$ & 8 (12.9\%) [4.6\%;21.2\%] & 5 (8.2\%) [1.3\%;15.1\%] & $(4.7 \%)[-6.1 \% ; 15.5 \%]$ & 0.559 \\
\hline Severe ${ }^{c}$ & $14(22.6 \%)$ [12.2\%;33.0\%] & 11 (18.0\%) [8.4\%;27.7\%] & $(4.6 \%)[-9.6 \% ; 18.7 \%]$ & 0.655 \\
\hline Severe and related ${ }^{c}$ & 7 (11.3\%) [3.4\%;19.2\%] & $2(3.3 \%)[-1.2 \% ; 7.7 \%]$ & $(8 \%)[-1.1 \% ; 17.1 \%]$ & 0.164 \\
\hline
\end{tabular}

Ankylosing Spondylitis Disease Activity Score including C-reactive protein (ASDAS-CRP) relapse was defined by increase $\geq 1.1$. Bath Ankylosing Spondylitis Disease Activity Index-visual analogue scale (BASDAI-VAS) was defined by increases of $20 \%$ or a 2 -unit increase in the $0-10$ scale. SER relapse was defined by BASDAI $\geq 4$, global clinical assessment by physician $\geq 4$ and at least one of three following criteria: patient assessment $\geq 4$, axial nocturnal pain (VAS) $\geq 4$, and increase in acute phase reactants (reactive ${ }^{\circ} \mathrm{C}$ protein (PCR) and/or erythrocyte sedimentation rate (ESR). Ankylosing Spondylitis Disease Activity Score-C reactive protein (ASDAS-C), which is calculated as $(0.12 \times$ back pain $)+(0.06 \times$ duration of morning stiffness $)+(0.11 \times$ patient GA $)+(0.07 \times$ peripheral pain/swelling $)+(0.58 \times \operatorname{Ln}(C R P+1))$; if CRP is not available but ESR is available, the last term is changed by $(0.29 \times \sqrt{ }(E S R))$. BASDAl is calculated as $\{A+B+C+D+[(E+F) / 2]\} / 5$ where $A$ to $E$ are 6 VAS, rated 0 (best) to 10 (worst) assessing (A) fatigue, (B) axial skeletal pain, (C) peripheral joint pain, (D) pain on contact or pressure, (E) intensity of morning stiffness and (F) duration of morning stiffness; VAS nocturnal axial pain is the patient's rating of nocturnal pain by VAS ranging from 0 (none) to 10 (worst). Patient Global Assessment (PGA) of disease activity was rated from 0 (best) to 10 (worst). Investigator's Global Assessment (IGA) of disease activity was rated from 0 (best) to 10 (worst). The Bath Ankylosing Spondylitis Functional Index (BASFI) is calculated as the average value of answers to 10 questions rated from 0 (best) to 10 (worst). Ankylosing Spondylitis Quality of Life (ASQoL - Spanish validated version) scores from 0 to 18, where lower scores indicate better health-related quality of life. Adverse event (AE) or infection presented as proportion $(95 \% \mathrm{Cl})$ of patients with at least one reported adverse event and/or infection; related presented as proportion $(95 \% \mathrm{Cl}$ ) of patients with at least one reported AE and/or infection assessed by the investigator as at least possibly related to TNF inhibitor (TNFi) treatment; severe presented as proportion $(95 \% \mathrm{Cl}$ ) of patients with at least one severe reported AE and/or infection according to standard definitions (fatal or life-threatening, required or prolonged the patient's hospitalization, caused significant or persistent disability, caused congenital anomaly/birth defect or required immediate medical intervention to avoid any of the previous outcomes); severe and related presented as proportion $(95 \% \mathrm{Cl})$ of patients with at least one reported severe and related AE and/or infection. SER: Spanish Society of Rheumatology (Sociedad Española de Reumatología)

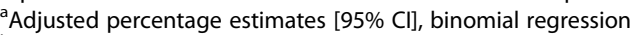

${ }^{\mathrm{b}}$ Adjusted least square means (standard error of the mean) [95\% Cl], mixed model for repeated measurements 'Number (\%) [95Cl]), analyzed by Fisher's exact test

One goal of personalized medicine is to identify probable beneficiaries of an intervention through clinical or biological markers [18]. We attempted to identify clinical and biological markers of a successful TNFi dose-reduction strategy. Baseline values of BASFI, ASQoL and the TNFi drug were associated with the odds of LDA after 1 year in univariate models; but in multivariate models only TNFi drug and BASFI remained significant. Similarly, hs-CRP was higher in subjects without LDA at 1 year. CRP best reflects objective inflammation and serum CRP is predictive of TNFi response in patients with AS [30].

Although reports have suggested no advantages of hs-CRP as compared with CRP in predicting the clinical response to TNFi [18], the results observed for hs-CRP are not unexpected, since it is a more sensitive technique. Reports have suggested a potential association with TNFi levels in rheumatoid arthritis, but data on AS are controversial [31-33]. We found no association between serum TNFi concentrations and the persistence of LDA at 1 year, although we had only few samples and these were for heterogeneous drugs. All samples had quantifiable TNFi levels, so none of the samples was tested for ADA. Other biomarkers, including calprotectin, did not significantly predict the likelihood of LDA at 1 year in our study. Recently, calprotectin levels have been suggested as a predictor of the clinical response [18], although in acute clinical response, and not in patients in remission. 
There were no interactions between dose regimen and TNFi or BASFI, indicating that their predictive value was independent of the actual dose regimen assigned, and multivariate models including biological markers were of low predictive value. Thus, we could not propose any marker to identify patients with higher chances of maintaining a low disease activity after reduction of TNFi dose.

Serious adverse events and infections were nominally less than one third in the reduced-dose group as compared to full dose, although not significant. This suggests low TNFi doses might have a better safety profile, but confirmation is needed, since the size and duration of our study were insufficient to draw conclusions. However, the differences might be relevant, especially since these are long-term treatments for often-fragile patients.

Our study has several limitations. First, due to limited funding and recruitment delays, the final sample size was smaller than anticipated. Although the sample was sufficient to conclude on the pre-determined primary objective, the limited sample size hindered the implementation of secondary objectives. Differences in adverse events were not significant and biological samples were obtained from only half the study population, likely reducing the ability to build a predictive model to identify patients with poorer chances of maintaining LDA after dose-halving. In addition, although the study was designed to include axSpA, only patients with AS were finally included. In addition, the study was not designed to determine which TNFi is more effective, nor powered to determine whether a specific TNFi can be reduced more successfully than another.

A conservative TNFi reduction schedule was applied in this study (about $50 \%$ of the recommended dose), and neither the potential benefit of an extra dose reduction or the effects of treatment withdrawal were studied. In clinical practice, infliximab can be reduced more than other TNFi, i.e., dose and interval; however, given the limited statistical power and the fact that this was an unplanned analysis, we cannot venture to show results of subgroup analysis by dose. Likewise, the 12-month follow up was insufficient to determine the potential effects of long-term TNFi reduction, especially on structural damage, which requires 2-4years to detect relevant changes [31,34]. A suggestion that TNFi dose tapering may be associated with more rapid radiographic progression than full doses regardless of LDA maintenance [31], especially in patients with baseline syndesmophytes, must be confirmed by prospective studies. Strict monitoring of radiographic progression of patients with AS is recommended if dose reduction is considered [31].

\section{Conclusion}

In spite of a number of limitations, this is the first randomized trial to support the non-inferiority of reduced-dose TNFi doses compared with full doses in patients with SpA. The results were consistent across many variables and TNFi drugs, and suggest a better safety profile with reduced doses, although the safety data must be interpreted with caution due to the small sample size. Overall, the study results support TNFi dose-reduction in patients with AS in stable clinical remission.

\section{Additional file}

Additional file 1: Table S1. Baseline characteristics, subset with inflammatory biomarkers. Table S2. Baseline predictors of loss of low disease activity at 1 year in the logistic regression analysis. Table S3. Univariate methods for loss of low disease activity at 1 year- subset with inflammatory biomarkers. Table S4. Listing of infectious adverse events. (DOCX $38 \mathrm{~kb}$ )

\section{Abbreviations}

AS: Ankylosing spondylitis; ASAS: Assessment of Spondyloarthritis International Society; ASDAS: Ankylosing Spondylitis Disease Activity Score; ASQoL: Ankylosing Spondylitis Quality of Life; axSpA: Axial spondyloarthritis; BASFI: Bath Anklylosing Spondylitis Functional Index; Cl: Confidence interval; CRP: C-reactive protein; DMARD: Disease-modifying anti-rheumatic drug; eCRF: Electronic case report form; ELISA: Enzyme-linked immunosorbent assay; FAS: Full analysis set; hs-CRP: High-sensitivity C-reactive protein; LDA: Low disease activity; NSAID: Non-steroidal anti-inflammatory drug; PPS: Per-protocol set; SD: Standard deviation; SpA: Spondyloarthritis; TNFi: TNF inhibitors

\section{Acknowledgements \\ REDES-TNF Investigators}

Miriam Almirall (IMIM - Hospital del Mar, Barcelona); Maria Aparicio (Hospital Universitari de Bellvitge, Barcelona); Agustí Sellas (Hospital Vall d'Hebron, Barcelona); Roser Vives, Nestor Albiñana; Mireia Moreno (Hospital Universitari ParcTaulí. I3PT. UAB. Sabadell (Barcelona); Teresa Clavaguera (Hospital Comarcal Palamós, Girona); Juan Carlos Torre-Alonso (Hospital Monte Naranco, Oviedo); Raúl Veroz (Hospital de Mérida, Mérida); Carlos RodríguezLozano (Hospital Universitario Dr. Negrín, Las Palmas de Gran Canaria); Luís Francisco Linares (Hospital Virgen de la Arrixaca, Murcia); Ana Urruticoechea (Hospital Can Misses, Eivissa, Baleares); Eduardo Collantes (Hospital Reina Sofía, Córdoba); Rosa María Morlà (Hospital de Sant Pau i Santa Tecla, Tarragona); Dèlia Reina (Hospital de Moises Broggi, Esplugues de Llobregat (Barcelona)); Eduardo Cuende (Hospital Príncipe de Asturias, Madrid); Pedro Zarco (Hospital Fundación Alcorcón, Madrid); Maria Cruz Fernández-Espartero (Hospital Universitario de Móstoles, Madrid); Rosario García-Vicuña (Hospital Universitario de La Princesa, Madrid); Carlos Alberto Montilla (Hospital Clínico de Salamanca, Salamanca); Alejandro Villalba, Dora Pascual (Hospital La Paz, Madrid); Cristina Campos (Hospital General de Valencia; Valencia); Antonio Juan (Hospital Son Llatzer; Mallorca); Rafael Ariza (Hospital Virgen Macarena; Sevilla); Consuelo Díaz-Miguel (Hospital Ramón y Cajal; Madrid); Manuel Maqueda (Hospital de Llerena, Llerena, Badajoz); Maria Pilar FernándezDapica (Hospital Doce de Octubre, Madrid); Manuel Fernández-Prada (Hospital Universitario de Guadalajara, Guadalajara); Enrique Batlle (Hospital Clinico de Sant Joan d'Alacant, Alacant); Carlos González-Fernández (Hospital Universitario Gregorio Marañón, Madrid); Rubén Queiro (Hospital

Universitario Central de Asturias; Oviedo).

\section{Funding}

The study was fully funded by grants from the Spanish Ministry of Health within the programme "Ayudas para el fomento de la investigación clínica independiente del Ministerio de Salud, Política Social e Igualdad - Orden SPI/ 2885/2011, de 20 de octubre" (project ID: EC11-229) and from the Ministerio de Economia y Consumo (MINECO)-Instituto de Salud Carlos III-Subdirección General de Evaluación and the European Fund for Regional Development (Project ID: PI13/02680). 


\section{Availability of data and materials}

The datasets supporting the conclusions of this article are available from the corresponding author on reasonable request.

\section{Authors' contributions}

All authors contributed to the design and the manuscript and FT performed the analysis. All authors read and approved the final manuscript.

\section{Ethics approval and consent to participate}

The trial was performed according to the study protocol and protocol amendments, which were approved by the Ethics Committee for Clinical Research of the participating sites and by the Spanish Agency for Medicines and Health Products, and complied with the ethical principles of biomedical research, Good Clinical Practice [35] and applicable Spanish legislation. Two amendments to the protocol reflected in the clinical trial registration sites were implemented after recruitment was initiated, which referred to inclusion of new investigators and recruiting sites, and to allow collection of blood samples for measurement of inflammatory mediators, drug plasma measurements and ADA.

\section{Consent for publication}

All authors have read and approved the final version and consent to publication.

\section{Competing interests}

No author has received any private funding, nor has any been influenced in the preparation of the manuscript. The following authors declare that they have no competing interests: CP, FT, CAS, AV and GC. In the past 2 years, JG has received grants to attend congresses and educational courses, has obtained fees for scientific consultancies and has received speaking fees and fees for participation in educational programmes from the following companies: Roche, MSD, Pfizer, AbbVie, Janssen Cilag, UCB Pharma, Novartis and Celgene. EdM has received grant/research support from AbbVie and Pfizer, honorarium as a speaker from Abbvie, MSD and Novartis and consultant fees from Abbvie and Novartis. JS has obtained grants to attend congresses, speaking fees and fees for participation in educational programmes from Abbvie, Pfizer, MSD, Roche, UCB and Menarini. XJ has attended advisory board meetings of Celgene, has received investigational grants from Abbvie and Pfizer and has received fees for participation in educational programmes from MSD, Pfizer and Abbvie. RS has received speaking fees and fees for attending advisory boards from Abbott/Abbvie, Bristol-Myers Squibb, MSD, Roche, UCB and Pfizer, investigational grants from Bristol-Myers Squibb, MSD, Roche, UCB, Pfizer, FER and SCR and funding for educational projects from MSD, Bristol-Myers Squibb and Abbvie.

\section{Publisher's Note}

Springer Nature remains neutral with regard to jurisdictional claims in published maps and institutional affiliations.

\footnotetext{
Author details

${ }^{1}$ Rheumatology Department, Hospital de Sabadell, Institut Universitari Parc Taulí, Universitat Autònoma de Barcelona, Sabadell, Barcelona, Spain. ${ }^{2}$ Clinical Pharmacology Unit, Hospital de Sabadell, Institut Universitari Parc Taulí, Universitat Autònoma de Barcelona, c/Taulí n¹, 08208 Sabadell, Barcelona, Spain. ${ }^{3}$ Rheumatology Department, Hospital Universitario de Bellvitge, Universitat de Barcelona, Bellvitge, Barcelona, Spain. ${ }^{4}$ Rheumatology Department, Hospital Universitario Puerta de Hierro- Majadahonda, Madrid, Spain. ${ }^{5}$ Medical Statistics core facility, IDIBAPS, Hospital Clínic, Biostatistics Unit, School of Medicine, Universitat Autònoma de Barcelona, Barcelona, Spain. ${ }^{6}$ Clinical Pharmacology Department, Hospital Puerta de Hierro Majadahonda, Madrid, Spain. ${ }^{7}$ Clinical Pharmacology Department, Hospital Universitario de Bellvitge - Universitat de Barcelona, Barcelona, Spain. ${ }^{8}$ Clinical Pharmacology Department, Hospital Clínic de Barcelona - Universitat de Barcelona, Barcelona, Spain. ${ }^{9}$ Rheumatology Department, Hospital Universitario La Paz, Madrid, Spain. ${ }^{10}$ Rheumatology Department, Hospital Clínic de Barcelona - Universitat de Barcelona, Barcelona, Spain.
}

Received: 1 August 2018 Accepted: 19 November 2018 Published online: 08 January 2019

\section{References}

1. Braun J, Bollow M, Remlinger G, Eggens U, Rudwaleit M, Distler A, Sieper J. Prevalence of spondylarthropathies in HLA-B27 positive and negative blood donors. Arthritis Rheum. 1998;41(1):58-67.

2. Gran JT, Husby G. The epidemiology of ankylosing spondylitis. Semin Arthritis Rheum. 1993;22(5):319-34.

3. Braun J, Pincus T. Mortality, course of disease and prognosis of patients with ankylosing spondylitis. Clin Exp Rheumatol. 2002;20(6 Suppl 28):S16-22.

4. Castillo-Ortiz JD, Ramiro S, Landewe R, van der Heijde D, Dougados M, van den Bosch F, Boonen A. Work outcome in patients with ankylosing spondylitis: results from a 12-year followup of an international study. Arthritis Care Res (Hoboken). 2016;68(4):544-52.

5. Smolen JS, Braun J, Dougados M, Emery P, Fitzgerald O, Helliwell P, Kavanaugh A, Kvien TK, Landewe R, Luger $T$, et al. Treating spondyloarthritis, including ankylosing spondylitis and psoriatic arthritis, to target: recommendations of an international task force. Ann Rheum Dis. 2014;73(1):6-16.

6. Zochling J, Bohl-Buhler MH, Baraliakos X, Feldtkeller E, Braun J. Nonsteroidal anti-inflammatory drug use in ankylosing spondylitis-a population-based survey. Clin Rheumatol. 2006;25(6):794-800.

7. Coates LC, Cawkwell LS, Ng NW, Bennett AN, Bryer DJ, Fraser AD, Emery P, Marzo-Ortega $\mathrm{H}$. Real life experience confirms sustained response to longterm biologics and switching in ankylosing spondylitis. Rheumatology (Oxford). 2008;47(6):897-900.

8. Bongartz T, Sutton AJ, Sweeting MJ, Buchan I, Matteson EL, Montori V. AntiTNF antibody therapy in rheumatoid arthritis and the risk of serious infections and malignancies: systematic review and meta-analysis of rare harmful effects in randomized controlled trials. JAMA. 2006;295(19):2275-85.

9. Busquets N, Carmona L, Suris X. Systematic review: safety and efficacy of anti-TNF in elderly patients. Reumatol Clin. 2011;7(2):104-12.

10. Dreyer L, Magyari M, Laursen B, Cordtz R, Sellebjerg F, Locht H. Risk of multiple sclerosis during tumour necrosis factor inhibitor treatment for arthritis: a population-based study from DANBIO and the Danish Multiple Sclerosis Registry. Ann Rheum Dis. 2016;75(4):785-6.

11. Baraliakos X, Listing J, Brandt J, Zink A, Alten R, Burmester G, Gromnica-Ihle E, Kellner H, Schneider M, Sorensen $\mathrm{H}$, et al. Clinical response to discontinuation of anti-TNF therapy in patients with ankylosing spondylitis after 3 years of continuous treatment with infliximab. Arthritis Res Ther. 2005;7(3):R439-44

12. Torrente V, Gratacos J, Juanola X, Sanmarti R, Suarez D, Moreno M. Infliximab withdrawal in patients with spondyloarthritis who presented criteria of clinical disease remission. an open study of clinical practise (REMINEA). Arthritis Rheum. 2009;60(Suppl 10):S1785.

13. Brandt J, Listing J, Haibel H, Sorensen H, Schwebig A, Rudwaleit M, Sieper J, Braun J. Long-term efficacy and safety of etanercept after readministration in patients with active ankylosing spondylitis. Rheumatology (Oxford). 2005; 44(3):342-8.

14. Navarro-Compan V, Moreira V, Ariza-Ariza R, Hernandez-Cruz B, VargasLebron C, Navarro-Sarabia F. Low doses of etanercept can be effective in ankylosing spondylitis patients who achieve remission of the disease. Clin Rheumatol. 2011;30(7):993-6.

15. Keeling S, Oswald A, Russell AS, Maksymowych WP. Prospective observational analysis of the efficacy and safety of low-dose (3 mg/kg) infliximab in ankylosing spondylitis: 4-year followup. J Rheumatol. 2006; 33(3):558-61.

16. Gonzalez-Alvaro I, Martinez-Fernandez C, Dorantes-Calderon B, Garcia-Vicuna R, Hernandez-Cruz B, Herrero-Ambrosio A, Ibarra-Barrueta O, Martin-Mola E, Monte-Boquet E, Morell-Baladron A, et al. Spanish Rheumatology Society and Hospital Pharmacy Society Consensus on recommendations for biologics optimization in patients with rheumatoid arthritis, ankylosing spondylitis and psoriatic arthritis. Rheumatology (Oxford). 2015;54(7):1200-9.

17. Chen DY, Chen YM, Hsieh TY, Hung WT, Hsieh CW, Chen HH, Tang KT, Lan $J$. Drug trough levels predict therapeutic responses to dose reduction of adalimumab for rheumatoid arthritis patients during 24 weeks of follow-up. Rheumatology (Oxford). 2016;55(1):143-8.

18. Maksymowych WP. Biomarkers in axial spondyloarthritis. Curr Opin Rheumatol. 2015;27(4):343-8. 
19. Rudwaleit M, van der Heijde D, Landewe R, Listing J, Akkoc N, Brandt J, Braun J, Chou CT, Collantes-Estevez E, Dougados M, et al. The development of Assessment of SpondyloArthritis international Society classification criteria for axial spondyloarthritis (part II): validation and final selection. Ann Rheum Dis. 2009;68(6):777-83.

20. Gossec L, Portier A, Landewe R, Etcheto A, Navarro-Compan V, Kroon F, van der Heijde D, Dougados M. Preliminary definitions of 'flare' in axial spondyloarthritis, based on pain, BASDAl and ASDAS-CRP: an ASAS initiative. Ann Rheum Dis. 2016;75(6):991-6.

21. Juanola Roura X, Zarco Montejo P, Sanz Sanz J, Munoz Fernandez S, Mulero Mendoza J, Linares Ferrando LF, Gratacos Masmitja J, Garcia de Vicuna R, Fernandez Carballido C, Collantes Estevez E, et al. Consensus Statement of the Spanish Society of Rheumatology on the management of biologic therapies in spondyloarthritis except for psoriatic arthritis. Reumatol Clin. 2011:7(2):113-23.

22. Pontes C, Gratacos J, Torres F, Avendano C, Sanz J, Vallano A, Juanola X, de Miguel E, Sanmarti R, Calvo G. Evaluation of dose reduction versus standard dosing for maintenance of remission in patients with spondyloarthritis and clinical remission with anti-TNF (REDES-TNF): study protocol for a randomized controlled trial. Trials. 2015;16:370.

23. Conti F, Ceccarelli F, Marocchi E, Magrini L, Spinelli FR, Spadaro A, Scrivo R, Valesini G. Switching tumour necrosis factor alpha antagonists in patients with ankylosing spondylitis and psoriatic arthritis: an observational study over a 5-year period. Ann Rheum Dis. 2007:66(10):1393-7.

24. Elashoff JD. nQuery Version 7.0 Advisor User's Guide. Statistical Solutions Ltd, Los Angeles, CA; 2007.

25. Newcombe RG. Interval estimation for the difference between independent proportions: comparison of eleven methods. Stat Med. 1998;17(8):873-90.

26. Lee J, Noh JW, Hwang JW, Oh JM, Kim H, Ahn JK, Lee YS, Cha HS, Koh EM. Extended dosing of etanercept $25 \mathrm{mg}$ can be effective in patients with ankylosing spondylitis: a retrospective analysis. Clin Rheumatol. 2010;29(10): 1149-54.

27. Zavada J, Uher M, Sisol K, Forejtova S, Jarosova K, Mann H, Vencovsky J, Pavelka K. A tailored approach to reduce dose of anti-TNF drugs may be equally effective, but substantially less costly than standard dosing in patients with ankylosing spondylitis over 1 year: a propensity scorematched cohort study. Ann Rheum Dis. 2016;75(1):96-102.

28. Pavy S, Brophy S, Calin A. Establishment of the minimum clinically important difference for the bath ankylosing spondylitis indices: a prospective study. J Rheumatol. 2005;32(1):80-5.

29. Kobelt G, Andlin-Sobocki P, Maksymowych WP. The cost-effectiveness of infliximab (Remicade) in the treatment of ankylosing spondylitis in Canada. J Rheumatol. 2006;33(4):732-40.

30. Ramiro S, van der Heijde D, van Tubergen A, Stolwijk C, Dougados M, van den Bosch F, Landewe R. Higher disease activity leads to more structural damage in the spine in ankylosing spondylitis: 12-year longitudinal data from the OASIS cohort. Ann Rheum Dis. 2014;73(8):1455-61.

31. Park JW, Kwon HM, Park JK, Choi JY, Lee EB, Song YW, Lee EY. Impact of dose tapering of tumor necrosis factor inhibitor on radiographic progression in ankylosing spondylitis. PLoS One. 2016;11(12):e0168958.

32. Bouman $C$, van Herwaarden $N$, van den Hoogen $F$, van der Maas $A$, van den Bemt $B$, den Broeder AA. Prediction of successful dose reduction or discontinuation of adalimumab, etanercept, or infliximab in rheumatoid arthritis patients using serum drug levels and antidrug antibody measurement. Expert Opin Drug Metab Toxicol. 2017;13(6):597-604.

33. Marsman AF, Kneepkens EL, Ruwaard J, Wei JC, Nurmohamed MT, van Denderen C, van der Horst-Bruinsma IE, Rispens T, Wolbink G. Search for a concentration-effect curve of adalimumab in ankylosing spondylitis patients. Scand J Rheumatol. 2016:45(4):331-4.

34. Poddubnyy D, Sieper J. Radiographic progression in ankylosing spondylitis/ axial spondyloarthritis: how fast and how clinically meaningful? Curr Opin Rheumatol. 2012;24(4):363-9.

35. International Conference on Harmonisation. ICH: E 6 (R1): Guideline for good clinical practice - Step 5. [https:/www.ich.org/fileadmin/Public_Web_ Site//CH_Products/Guidelines/Efficacy/E6/E6_R1_Guideline.pdf]. Last access: $30^{\text {th }}$ November 2018

\section{Ready to submit your research? Choose BMC and benefit from:}

- fast, convenient online submission

- thorough peer review by experienced researchers in your field

- rapid publication on acceptance

- support for research data, including large and complex data types

- gold Open Access which fosters wider collaboration and increased citations

- maximum visibility for your research: over $100 \mathrm{M}$ website views per year

At BMC, research is always in progress.

Learn more biomedcentral.com/submissions 